\title{
铜催化芳嗍酸与亚磷酸酯在空气下的有氧碳-磷氧化偶联反应
}

\author{
徐 清*, $a$ 贾小娟 ${ }^{a}$ 李晓慧 ${ }^{a}$ 孙 清 ${ }^{a}$ \\ 周永波 ${ }^{b}$ 尹双凤 ${ }^{b}$ 韩立彪*, $b, c$ \\ ( ${ }^{a}$ 温州大学化学与材料工程学院 温州 325035) \\ ( ${ }^{b}$ 湖南大学化学化工学院 长沙 410082) \\ (c产业技术综合研究所(AIST) 筑波 茨城 305-8565 日本)
}

\begin{abstract}
摘要 在 $\mathrm{Cu}(\mathrm{OAc})_{2} / \mathrm{TMEDA}$ 体系催化下, 芳蹶酸与亚磷酸二乙酯可在室温下以空气为直接氧化剂通过氧化偶联反应生 成芳基膦酸酯化合物. 该反应使用较为廉价低毒的铜盐为催化剂, 不怕水、不怕氧, 底物适用范围较广, 目标产物选择 性高、收率良好. 因此，与已知金属催化卤代芳烃的碳一磷偶联反应相比是一种相对温和高效的合成芳基膦酸酯的方法. 关键词 铜催化; 芳嗍酸; 亚磷酸酯; 氧化偶联; 碳一磷键构建; 芳基膦酸酯
\end{abstract}

\section{Copper-Catalyzed Aerobic Oxidative C-P Coupling of Aryl- boronic Acids and Diethyl Phosphite under Air}

\author{
$\mathrm{Xu}$, Qing $^{*, a} \quad \mathrm{Jia}, \mathrm{Xiaojuan}^{a}$ \\ Li, Xiaohui ${ }^{a}$ \\ Sun, Qing ${ }^{a}$ \\ Zhou, Yongbo $^{b} \quad$ Yin, Shuangfeng $^{b} \quad$ Han, Libiao*,b $^{*}$ \\ ( ${ }^{a}$ College of Chemistry and Materials Engineering, Wenzhou University, Wenzhou 325035) \\ $\left({ }^{b}\right.$ College of Chemistry and Chemical Engineering, Hunan University, Changsha 410082) \\ ( ${ }^{c}$ National Institute of Advanced Industrial Science and Technology (AIST), Tsukuba, Ibaraki 305-8565, Japan)
}

\begin{abstract}
By using $\mathrm{Cu}(\mathrm{OAc})_{2} / \mathrm{TMEDA}$ as the catalyst and air as the direct oxidant, arylboronic acids and diethyl phosphite can readily undergo an aerobic oxidative $\mathrm{C}-\mathrm{P}$ coupling reaction under air at room temperature to give the useful arylphosphonates. This reaction uses relatively cheaper and less toxic copper salts as the catalyst, tolerates moisture and aerobic conditions. is suitable for a comparatively broad scope of substrates, and can give the target arylphosphonates in high selectivities and good yields. In comparison with conventional metal-catalyzed $\mathrm{C}-\mathrm{P}$ coupling reactions of aryl halides, it is a relatively mild and efficient method for the synthesis of arylphosphonates.

Keywords copper catalysis; arylboronic acids; diethyl phosphite; oxidative coupling; C-P bond construction; arylphosphonates
\end{abstract}

有机膦化合物在有机合成、催化、材料、生化和医 药研究等多方面均具有广泛的合成与应用用途 ${ }^{[1]}$, 因此, 有机膦化合物的合成方法研究一直受得到人们的关注. 通过过渡金属催化方法将易得易制备的磷-氢化合物导 入碳链是构建碳一磷键合成有机膦化合物最重要的手段 之一. 目前, 相对成熟的方法主要有磷-氢键对碳-碳不 饱和键的磷-氢加成反应 ${ }^{[2]}$ 和碱存在下磷-氢化合物与卤 代芳烃的碳-磷偶联反应 ${ }^{[3]}$. 近年来, 通过磷-氢化合物
与杂原子-氢或碳-氢化合物在过渡金属催化下的脱氢、 氧化、碳一氢活化等方式的直接碳一磷偶联反应也逐渐出 现报道 ${ }^{[2 \mathrm{c}, 4]}$, 成为构建碳一磷键合成有机膦化合物的新兴 研究方向. 但碳-氢活化及其碳-磷偶联等反应 ${ }^{[2 c, 4]}$ 目前 仍存在不少局限性, 对于活性较低或缺乏导向基团的 碳一氢键的活化还很难实现，因此开发可以替代卤代芳 烃偶联反应 ${ }^{[3]}$ 的新方法也是一种很好的策略.

另一方面，与使用卤代芳烃为底物的常规偶联方法

\footnotetext{
*E-mail: qing-xu@wzu.edu.cn, libiaohan@yahoo.com

Received March 23, 2014; revised May 10, 2014; published online May 23, 2014.

Project supported by the National Natural Science Foundation of China (No. 20902070) and the Natural Science Foundation of Zhejiang Province for Distinguished Young Scholars (No. LR14B020002).

国家自然科学基金(No. 20902070)和浙江省自然科学杰出青年基金(No. LR14B020002)资助项目.
} 
相比，以芳硼酸为芳基供体的 Suzuki 偶联反应有更多的 优点, 至今已有极其广泛的研究和应用 ${ }^{[5]}$. 与毒性较大 的卤代芳烃特别是活性较高的溴代和碘代芳烃相比, 芳 嗍酸毒性低、不怕水、不怕氧, 其参与的很多反应无需 惰性气体保护, 且适用于含活泼敏感官能团的底物, 从 而可大大简化反应条件和操作，同时扩展了反应的官能 团兼容性和底物的适用范围 ${ }^{[5]} .1998$ 年, Chan, Lam 和 Evans 等小组 ${ }^{[6]}$ 相继报道了铜作用下胺、酰胺和氮杂环 的氮一氢键和酚的氧一氢键与芳嗍酸的碳一氧和碳 - 氮偶 联反应合成胺、酰胺、杂环和醚衍生物的新方法. 受到 这些报道的启发, 此后更多的研究小组都加入了过渡金 属作用或催化芳硼酸与杂原子-氢化合物的碳-杂原子 偶联反应研究, 相继报道了很多相关的偶联方法 ${ }^{[\mathrm{d} \sim 5 \mathrm{f}, 7]}$. 不仅是常规的杂原子-氢化合物的反应可以顺利地参与 构建碳一氮、碳一氧、碳一硫键, 某些方法还可以使用二硫 醚、二硒醚、二碲醚或零价硫属单质为起始原料实现直 接高效的偶联反应 ${ }^{[\mathrm{d} \sim 5 \mathrm{f}, 7 \mathrm{~g} \sim 7 \mathrm{~h}]}$. 相比之下, 芳硼酸与磷氢化合物的碳一磷偶联反应研究很少. 据我们所知, 直 到 2009 年才由 Larhed 小组 ${ }^{[8]}$ 首次报道了微波辐射促进 下、钯催化芳硼酸与亚磷酸酯的碳-磷偶联反应. 接着, Zhao 小组 ${ }^{[9]}$ 报道了使用更为廉价的铜和镍为催化剂的 碳一磷偶联方法. 鉴于芳硼酸作为芳基化试剂的优点 ${ }^{[5]}$ 以及铜催化方法在偶联反应中的诸多优势和广泛应 用 ${ }^{[10,11]}$, 在我们组有机膦化合物合成方法 ${ }^{[2,4]}$ 和铜催化 氧化反应 ${ }^{[12]}$ 的研究过程中, 我们也设想, 通过铜催化芳 硼酸与磷-氢化合物的氧化偶联反应, 可能是实现碳一磷 键构建合成有机膦化合物的一种良好方法 ${ }^{[13]}$. 本文我 们将报道 $\mathrm{Cu}(\mathrm{OAc})_{2} / \mathrm{TMEDA}$ 催化芳硼酸与亚磷酸二乙
酯在室温空气下直接氧化偶联生成芳基膦酸酯化合物 的方法.

\section{1 结果与讨论}

如表 1 所示, 我们以苯硼酸(1a)与二乙基亚磷酸酯 (2a) 的反应为模型来优化条件. 我们首先选用 $\mathrm{Cu}(\mathrm{OAc})_{2}$ (5 mol\%) 为催化剂, 直接购买的甲苯 $(0.5 \mathrm{~mL})$ 为溶剂, 无配体, 无碱, 室温 $\left(25 \sim 30{ }^{\circ} \mathrm{C}\right)$, 空气等条件进行尝试. 反应 $24 \mathrm{~h}$ 后，通过 GC-MS 检测到了少量目标产物苯基 膦酸二乙酯 3a (Entry 1). 在上述条件下, 我们初步尝试 了 DMSO，THF，1４-二氧六环等几种常用的有机溶剂, 发现无反应或只有少量产物生成, 其中 $\mathrm{CH}_{2} \mathrm{Cl}_{2}$ 的效果 较好(Entry 1). 文献调研显示铜催化和芳硼酸的偶联反 应中, 配体和碱可能起到重要的作用 ${ }^{[5,10,14]}$, 因此我们 继续使用 $\mathrm{CH}_{2} \mathrm{Cl}_{2}$ 为溶剂对配体和碱作用进行了考察. 当上述反应中只加入配体如 $N, N, N^{\prime}, N^{\prime}$-四甲基乙二胺 (TMEDA)后, 产物稍有增加, 但是产率仍然很低(Entry $2)$; 只加入碱如 $\mathrm{K}_{2} \mathrm{CO}_{3}$ 时, 产物明显增加, 但同时也生 成不少的连二磷酸酯副产物(Entry 3). 这大概是由于在 空气氛围下, 连二磷酸酯容易在铜催化下由亚磷酸酯的 自身氧化偶联反应生成 ${ }^{[4 \mathrm{~d}]}$. 因此, 为避免连二磷酸酯的 大量产生，同时为提高目标偶联反应的选择性和产率， 我们对催化剂体系的各个参数进行了仔细的优化. 进一 步研究表明, 反应同时使用铜催化剂、配体和碱可大大 提高目标产物 3a 的选择性和产率(Entry 4). 但是, 只加 配体和碱，不加铜催化剂时反应也不会发生(Entry 5), 这也表明铜催化剂在反应中的关键作用. 如果反应在氮

表 1 铜催化苯嗍酸与亚磷酸二乙酯偶联反应条件篮选 ${ }^{a}$

Table 1 Condition screening for Cu-catalyzed coupling reaction of phenylboronic acid and diethyl phosphite

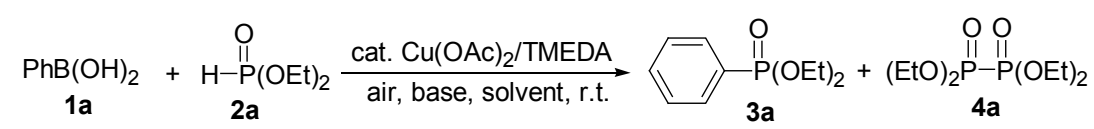

\begin{tabular}{cclcccc}
\hline Entry & $\mathrm{Cu}(\mathrm{OAc})_{2} / \mathrm{TMEDA}(\mathrm{mol} \%)$ & Base (equiv.) & Solvent & Time/h & $\mathbf{3 a}^{b} / \%$ & $\mathbf{3 a}^{2} / \mathbf{4 a}$ \\
\hline 1 & 5 & - & $\mathrm{Solvents}^{c}$ & 24 & Trace & - \\
2 & $5(10)$ & - & $\mathrm{CH}_{2} \mathrm{Cl}_{2}$ & 24 & Trace & - \\
3 & 5 & $\mathrm{~K}_{2} \mathrm{CO}_{3}(1)$ & $\mathrm{CH}_{2} \mathrm{Cl}_{2}$ & 24 & 23 & $47 / 53$ \\
4 & $5(10)$ & $\mathrm{K}_{2} \mathrm{CO}_{3}(1)$ & $\mathrm{CH}_{2} \mathrm{Cl}_{2}$ & 24 & 81 & $83 / 17$ \\
5 & $(10)$ & $\mathrm{K}_{2} \mathrm{CO}_{3}(1)$ & $\mathrm{CH}_{2} \mathrm{Cl}_{2}$ & 24 & - & - \\
$6^{e}$ & $5(10)$ & $\mathrm{K}_{2} \mathrm{CO}_{3}(1)$ & $\mathrm{CH}_{2} \mathrm{Cl}_{2}$ & 24 & Trace & - \\
7 & $5(10)$ & $\mathrm{Cs}_{2} \mathrm{CO}_{3}(1)$ & $\mathrm{CH}_{2} \mathrm{Cl}_{2}$ & 24 & $90(79)$ & $90 / 10$ \\
8 & $5(10)$ & $\mathrm{Cs}_{2} \mathrm{CO}_{3}(0.6)$ & $\mathrm{CH}_{2} \mathrm{Cl}_{2}$ & 12 & 90 & $93 / 7$ \\
9 & $5(10)$ & $\mathrm{Cs}_{2} \mathrm{CO}_{3}(0.6)$ & $\mathrm{CH}_{3} \mathrm{CN}$ & 12 & $97(86)$ & $97 / 3$ \\
10 & $2(4)$ & $\mathrm{Cs}_{2} \mathrm{CO}_{3}(0.6)$ & $\mathrm{CH}_{3} \mathrm{CN}$ & 12 & $95(85)$ & $97 / 3$ \\
\hline
\end{tabular}

${ }^{a}$ The mixture of $\mathrm{PhB}(\mathrm{OH})_{2}(1.5 \mathrm{mmol}, 1.5$ equiv. $), \mathrm{HP}(\mathrm{O})(\mathrm{OEt})_{2}(1.0 \mathrm{mmol}), \mathrm{Cu}(\mathrm{OAc})_{2} \cdot \mathrm{H}_{2} \mathrm{O}$, ligand, and a base in a solvent $(0.5 \mathrm{~mL})$ was stirred at room temperature $\left(25 \sim 30{ }^{\circ} \mathrm{C}\right)$ in a Schlenk tube equipped with an air balloon. The reaction was then monitored by TLC and/or GC-MS. ${ }^{b}$ GC yields (isolated yields in parenthesis) based on 2a. ${ }^{c}$ Ratios determined by GC-MS analysis. ${ }^{d}$ The usual solvents (toluene, DMSO, THF, dioxane, $\mathrm{CH}_{2} \mathrm{Cl}_{2}$, etc.) were tested. ${ }^{e}$ Reaction performed under $\mathrm{N}_{2}$. 
气保护下进行, 则只能观察到少量产物(Entry 6), 也说 明空气在反应中起很大的作用, 极有可能是其中的氧气

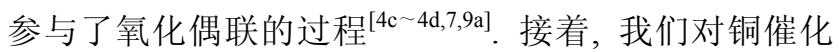
剂、配体、碱、溶剂的种类及其用量继续进行优化. 我 们意识到, 碱在过渡金属催化反应中起重要的作用 ${ }^{[14]}$, 因此考察了各类不同的无机碱如碳酸盐 $\mathrm{M}_{2} \mathrm{CO}_{3}(\mathrm{M}=\mathrm{Li}$, $\mathrm{Na}, \mathrm{Cs}, 1$ equiv.)、苛性碱 $\mathrm{MOH}(\mathrm{M}=\mathrm{Li}, \mathrm{Na}, \mathrm{K}, \mathrm{Cs}, 2$ equiv.)、 $t$ - $\mathrm{BuOK}$ (2 equiv.) 等, 得到选择性和产率高低不 等的产物和副产物; 其中弱碱性的 $\mathrm{Cs}_{2} \mathrm{CO}_{3}$ 的效果较好, 可得到相对高产和高选择的目标产物(Entry 7). 使用 $\mathrm{Cs}_{2} \mathrm{CO}_{3}$ 为碱时, 不仅用量可减少到 0.6 equiv., 反应时间 也可缩短到 $12 \mathrm{~h}$, 但仍然保持很好的反应效果和产物收 率(Entry 8). 使用更少量的 $\mathrm{Cs}_{2} \mathrm{CO}_{3}$ (如 0.5 equiv.) 则导致 反应时间延长、产物收率下降. 在此较优的条件下, 我 们再次对溶剂进行考察, 发现乙腈是最好的溶剂, 可得 到高选择和高产率的产物(Entry 9). 我们继续对常用的 铜催化剂 $\left(\mathrm{CuCl}_{2}, \mathrm{CuBr}_{2}, \mathrm{Cu}\left(\mathrm{NO}_{3}\right)_{2}, \mathrm{CuSO}_{4}, \mathrm{Cu}\left(\mathrm{CF}_{3} \mathrm{C}-\right.\right.$ $\mathrm{OO})_{2}, \mathrm{CuCl}, \mathrm{CuBr}, \mathrm{CuI}, \mathrm{CuCN}$ 等) 和常见配体 $\left(\mathrm{PPh}_{3}\right.$, $\mathrm{Et}_{3} \mathrm{~N}$, 吡啶、联吡啶、 $N, N$-二甲基乙二胺等)进行了考察, 发现 $\mathrm{Cu}(\mathrm{OAc})_{2}$ 和 TMEDA 仍是较好的催化剂和配体. 我 们还对苯嘲酸、催化剂和配体的用量进行了进一步考察. $\mathrm{Cu}(\mathrm{OAc})_{2}$ 和 TMEDA 的用量分别可降低到 2 和 $4 \mathrm{~mol} \%$, 几乎不影响反应效率，最终可得高选择且 $85 \%$ 良好分离 收率的产物(Entry 10). 虽然过多的苯喼酸对反应没有 明显的促进作用, 但过少的苯硼酸则可导致反应不完 全、产物收率降低, 这可能是由于苯硼酸容易发生自身 偶联产生联苯副产物 ${ }^{[15]}$, 从而消耗了了一部分苯硼酸 的缘故.

优化得到最佳的反应条件后(表 1, Entry 10), 我们 对不同的底物进行了考察以扩展该方法的适用范围. 如 表 2 所示, 与无取代的苯嗍酸类似, 含推电子基团如甲 氧基、甲基(Entries 2 7)和含吸电子基团如卤素、三氟 甲基、腈基、羰基(Entries 8 14)的芳嗍酸都可以与亚磷 酸二乙酯反应以中等到良好收率得到相应的芳基膦酸 二乙酯. 一些位阻较大的芳嗍酸如邻位取代芳砋酸和 1萗硼酸大概受到取代基空间位阻上的影响，在产物收率 上有所降低(Entries 4, 7, 11, 15). 在优化的反应条件下, 一些较为活泼的取代基如卤素、腈基和羰基都在以上反 应中都得到了保留(Entries $8 \sim 11,13,14)$, 而不会发生 与芳嗍酸的偶联或加成等文献报道上存在的可能的竞 争反应 ${ }^{[5,16]}$. 这大概是因为当前的反应条件(室温、空气、 弱碱条件)比较温和的缘故，也显示了该方法的较广的 官能团兼容性和对芳硼酸与磷-氢键碳一磷偶联反应的 高选择性. 保留这些较活泼的官能团后, 还可在以后的 反应中进一步利用, 实现多步合成的目的. 此外, 我们
表 2 空气下铜催化芳嗍酸与亚磷酸酯的氧化偶联反应 ${ }^{a}$

Table 2 Copper-catalyzed oxidative coupling of arylboronic acids and diethyl phosphite under air
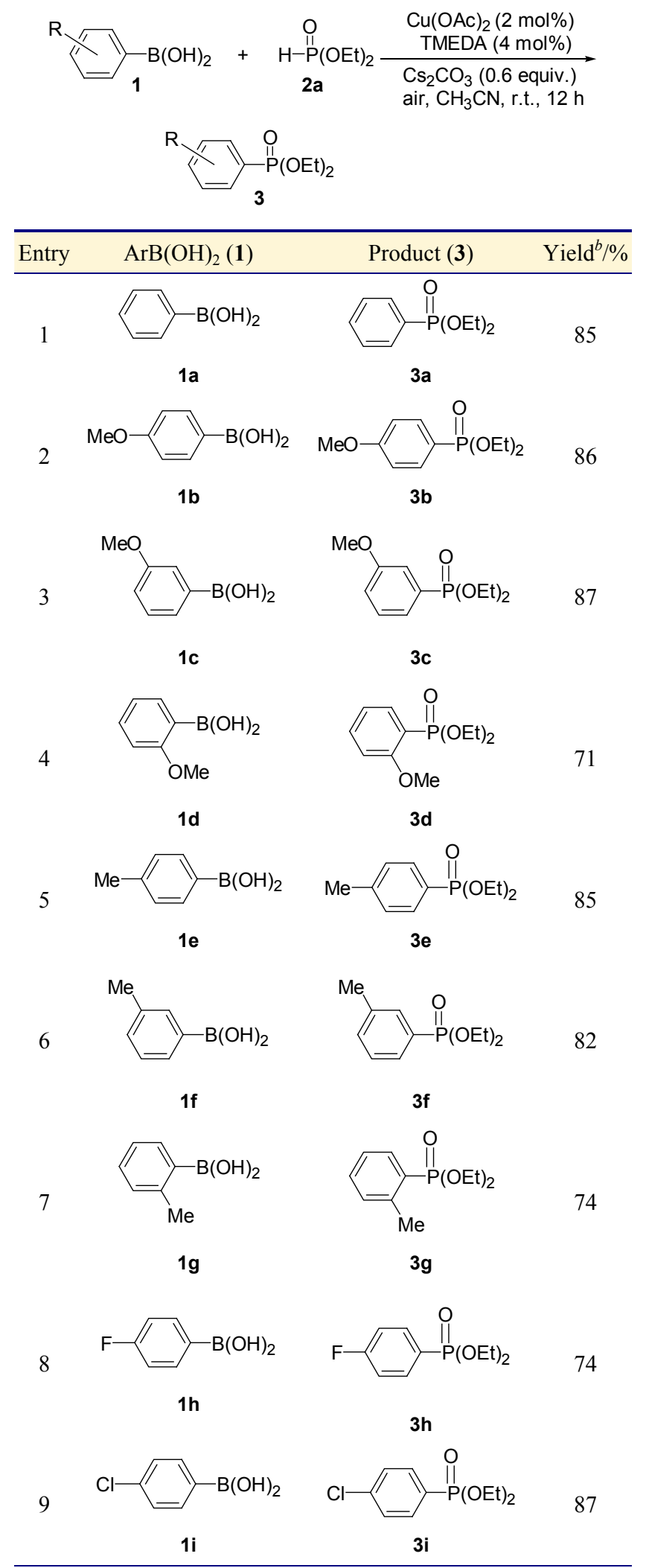
续表

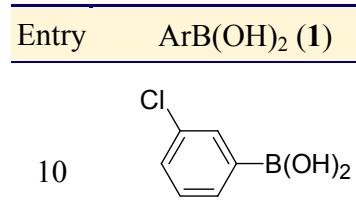

1j

11<smiles>Oc1ccccc1Cl</smiles>

1k

12<smiles>O[Ga]c1ccc(C(F)(F)F)cc1</smiles>

11

13<smiles>N#Cc1ccc(Br)cc1</smiles>

$1 \mathrm{~m}$

14<smiles>CC(=O)c1ccc(Br)cc1</smiles>

1n<smiles>O[Ga]c1cccc2ccccc12</smiles>

10<smiles>O[Bi]c1ccco1</smiles>

$1 p$

17<smiles>O[Bi]c1cccs1</smiles>

$1 q$

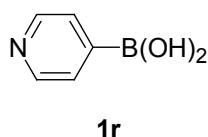

Product (3) Yield $^{b} / \%$

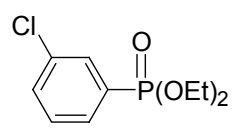

86

3j<smiles>CCOP(=O)(OCC)c1ccccc1Cl</smiles>

74<smiles>CCOP(=O)(OCC)c1ccc(C(F)(F)F)cc1</smiles><smiles>CCOP(=O)(OCC)c1ccc(C#N)cc1</smiles><smiles>CCOP(=O)(OCC)c1ccc(C(C)=O)cc1</smiles><smiles>CCO[PH](=O)c1cccc2ccccc12</smiles>
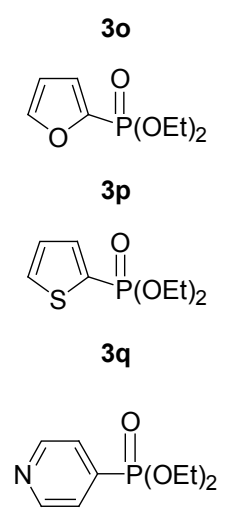

31

$3 n$

$3 p$

$3 q$

84

82
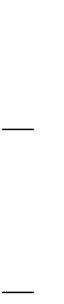

\section{$3 r$}

芳硼酸的偶联和氧化偶联反应 ${ }^{\left[{ }^{[-7]}\right.}$ 以及磷-氢化合物参 与氧化偶联反应 ${ }^{[4]}$ 的机理, 我们提出了 $\mathbf{A}$ 和 $\mathbf{B}$ 两种可能 的循环机理(Scheme 1). 首先氮配体与二价铜盐 $\mathrm{CuX}_{2}$ $(\mathrm{X}=\mathrm{OAc}$ ，卤素等配位阴离子)形成四配位的络合物 $\mathbf{5}$; 在碱的作用下，一般占主要组成的五价亚磷酸酯异构体 $\mathbf{2 a}$ 异构化成配位能力更强的三价亚磷酸酯 $\mathbf{2} \mathbf{a}^{\prime} ; \mathbf{5}$ 和 $\mathbf{2 a}^{\prime}$ 作用生成络合物 $\mathbf{6}$. 在某些条件下, 6 可能与 $\mathbf{2} \mathrm{a}^{\prime}$ 继续反 应生成副产物 $\mathbf{4} \mathrm{a}^{[\mathrm{dd}]}$; 在优化的条件下, 该副反应可得到 控制, 反应主要按如下的目标交叉偶联反应进行. 接着, 芳硼酸与络合物 6 发生转金属反应形成络合物 7, 同时 消除硼酸衍生物. 7 发生消除反应实现碳一磷键的偶联得 到目标产物芳基磷酸酯 $\mathbf{3}$, 并释放出一种铜络合物. 由 于铜络合物与氧分子的结合能力很强 ${ }^{[11]}$, 在空气存在 下形成铜一氧络合物 $8^{[5 \sim 7,11]}$, 铜就被氧化重新生成络合 物 $\mathbf{5}$, 从而完成 $\mathbf{A}$ 催化循环. 由于单独的芳硼酸能与铜 催化剂进行转金属反应可生成芳硼酸自身氧化偶联的 联芳基副产物 ${ }^{[15]}$, 而且发现反应中需使用过量的芳硼 酸以实现好的反应效果, 因此在反应机理中, 络合物 $\mathbf{5}$ 与芳硼酸先作用形成络合物 $\mathbf{6}^{\prime}$, 再与 $\mathbf{2} \mathrm{a}^{\prime}$ 作用生成 7 , 最 后生成产物 $\mathbf{3}$ 和再生铜催化剂的催化循环 $\mathbf{B}$ 也是可能存 在的.

\section{2 结论}

我们研究了室温、空气下铜催化芳硼酸与亚磷酸酯 的氧化偶联反应，开发了一种构建碳一磷键简便合成芳 基膦酸酯的方法. 该方法使用较低用量的廉价低毒的铜 催化剂, 在室温下直接使用空气为氧化剂, 反应条件简 单、温和、经济, 对底物活泼官能团的兼容性好, 因此 是一种相对较好的芳基膦酸酯的合成方法, 也进一步拓 展了铜催化碳一杂原子氧化偶联反应和碳-磷键构建的 合成方法.

\section{3 实验部分}

\section{1 仪器与试剂}

所用催化剂、药品和溶剂均为市售分析纯或化学纯 试剂, 除特别说明外, 未经处理直接使用. 亚磷酸二乙 酯经减压蒸馏纯化后使用 $\left(72 \sim 74{ }^{\circ} \mathrm{C} / 1067 \mathrm{~Pa}\right)$. 反应在 管型反应器中进行, 通过薄层层析(TLC)或气相-质谱联 用(GC-MS)监测反应进度. 产物采用柱层析方法使用石 油醚/乙酸乙酯为淋洗剂纯化. 核磁氢谱 $(500 \mathrm{MHz}$ ) 和磷 谱(202 MHz)使用 Bruker Avance-III 500 仪器测定, 以氛 代氯仿为溶剂、TMS 或 $85 \%$ 磷酸気代水溶液为内标; 质 谱和气相一质谱联用(GC-MS)Shimadzu GCMS-QP2010 Plus 质谱仪(日本岛津公司)测定. 现象和规律, 我们认为与芳硼酸的其他碳-杂原子构建 的氧化偶联反应机理类似 ${ }^{[5 \mathrm{~d} \sim 5 \mathrm{f}, 6,7]}$. 参考过渡金属催化 


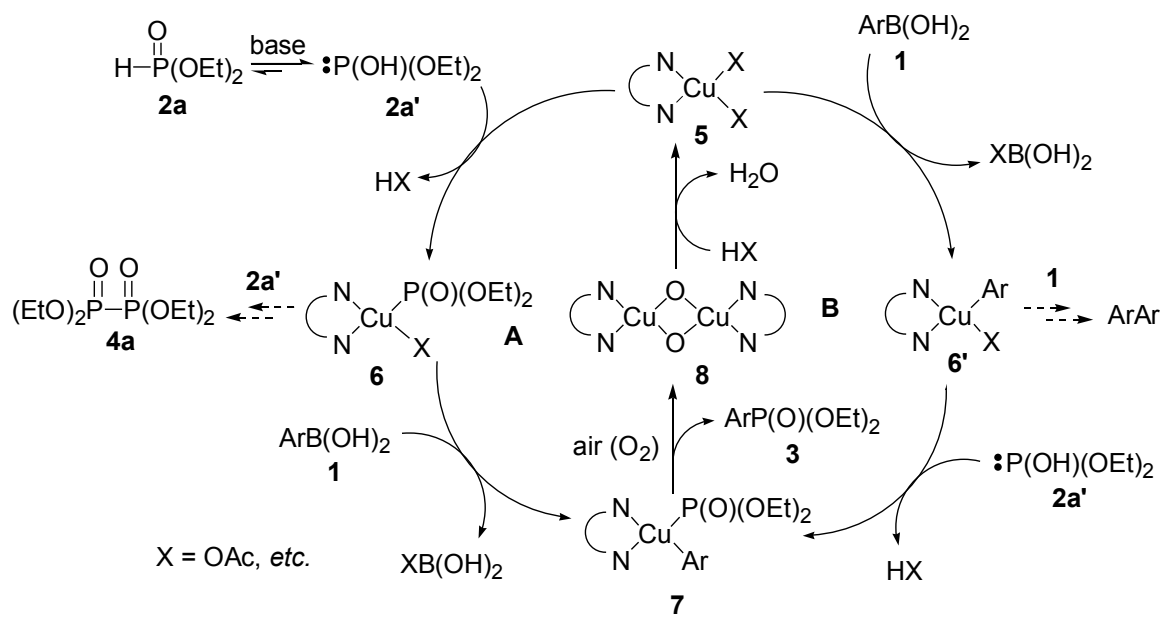

Scheme 1

\section{2 芳基膦酸酯的典型合成步骤}

空气下往带支口的管形中加入苯硼酸(1a) $(0.182 \mathrm{~g}$, $1.5 \mathrm{mmol})$ 、亚磷酸二乙酯 $(2 \mathrm{a})(0.1380 \mathrm{~g}, 1.0 \mathrm{mmol})$ 、一 水合醋酸铜 $(0.004 \mathrm{~g}, 0.02 \mathrm{mmol}, 2 \mathrm{~mol} \%)$ 、TMEDA $(0.0046 \mathrm{~g}, 0.04 \mathrm{mmol}, 4 \mathrm{~mol} \%)$ 、碳酸铯 $(0.1955 \mathrm{~g}, 0.6$ $\mathrm{mmol}, 60 \mathrm{~mol} \%)$ 和乙腈溶剂 $(0.5 \mathrm{~mL})$, 支口配上充满空 气的气球, 然后在室温下(约 $25 \sim 30{ }^{\circ} \mathrm{C}$ )搅拌, TLC 和 GC-MS 监测反应，反应 $12 \mathrm{~h}$ 后基本完全. 混合物用柱层 析分离纯化(淋洗剂: 石油醚和乙酸乙酯), 最后以 $85 \%$ 分离收率得到苯基膦酸二乙酯. 其它芳基膦酸酯用类似 方法合成，已知化合物图谱经与已知文献数据核对一 致.

苯基膦酸二乙酯(3a) $)^{[8]}$ : 无色油状物. ${ }^{1} \mathrm{H}$ NMR (500 $\left.\mathrm{MHz}, \mathrm{CDCl}_{3}\right) \delta: 7.83 \sim 7.79(\mathrm{~m}, 2 \mathrm{H}), 7.58 \sim 7.54(\mathrm{~m}, 1 \mathrm{H})$, $7.49 \sim 7.45(\mathrm{~m}, 2 \mathrm{H}), 4.19 \sim 4.04(\mathrm{~m}, 4 \mathrm{H}), 1.32(\mathrm{t}, J=7.0$ $\mathrm{Hz}, 6 \mathrm{H}) ;{ }^{31} \mathrm{P} \mathrm{NMR}\left(202 \mathrm{MHz}, \mathrm{CDCl}_{3}\right.$ ) $\delta: 18.9$. MS (EI) $\mathrm{m} / z$ (\%): 214 (25), 186 (14), 158 (91), 141 (88), 105 (48), 94 (44), 77 (100), 65 (13), 51 (37).

(4-甲氧基苯基)膦酸二乙酯 $(\mathbf{3 b})^{[17]}$ : 无色油状物. ${ }^{1} \mathrm{H}$ NMR (500 MHz, $\left.\mathrm{CDCl}_{3}\right) \delta: 8.17(\mathrm{~d}, J=8.0 \mathrm{~Hz}, 2 \mathrm{H})$, $7.02(\mathrm{~d}, J=8.0 \mathrm{~Hz}, 2 \mathrm{H}), 4.26 \sim 4.17(\mathrm{~m}, 4 \mathrm{H}), 3.90(\mathrm{~s}$, $3 \mathrm{H}), 1.35(\mathrm{t}, J=7.0 \mathrm{~Hz}, 6 \mathrm{H}) ;{ }^{31} \mathrm{P}$ NMR $(202 \mathrm{MHz}$, $\mathrm{CDCl}_{3}$ ) $\delta: 19.8$; MS (EI) $m / z$ (\%): 245 (4), 244 (35), 216 (49), 188 (100), 171 (49), 135 (33), 123 (20), 108 (94), 77 (29), 65 (15).

(3-甲氧基苯基)膦酸二乙 $(3 \mathbf{c})^{[18]}$ : 无色油状物. ${ }^{1} \mathrm{H}$ NMR $\left(500 \mathrm{MHz}, \mathrm{CDCl}_{3}\right) \delta: 7.40 \sim 7.35(\mathrm{~m}, 3 \mathrm{H}), 7.10 \sim$ $7.08(\mathrm{~m}, 1 \mathrm{H}), 4.19 \sim 4.04(\mathrm{~m}, 4 \mathrm{H}), 3.85(\mathrm{~s}, 3 \mathrm{H}), 1.33(\mathrm{t}$, $J=7.0 \mathrm{~Hz}, 6 \mathrm{H}) ;{ }^{31} \mathrm{P}$ NMR $\left(202 \mathrm{MHz}, \mathrm{CDCl}_{3}\right) \delta: 18.7$. MS (EI) $m / z$ (\%): 245 (7), 244 (59), 216 (45), 188 (28), 172 (77), 135 (35), 108 (100), 92 (28), 77 (44), 65 (18).
(2-甲氧基苯基)膦酸二乙酯 (3d) ${ }^{[19]}$ : 无色油状物. ${ }^{1} \mathrm{H}$ NMR $\left(500 \mathrm{MHz}, \mathrm{CDCl}_{3}\right) \delta: 7.30 \sim 7.26(\mathrm{~m}, 1 \mathrm{H})$, $7.14 \sim 7.11(\mathrm{~m}, 1 \mathrm{H}), 6.96 \sim 6.89(\mathrm{~m}, 2 \mathrm{H}), 4.28 \sim 4.21(\mathrm{~m}$, $4 \mathrm{H}), 3.87(\mathrm{~s}, 3 \mathrm{H}), 1.36(\mathrm{td}, J=7.0,1.0 \mathrm{~Hz}, 6 \mathrm{H}) ;{ }^{31} \mathrm{P}$ NMR (202 MHz, $\left.\mathrm{CDCl}_{3}\right) \delta: 19.7$; MS (EI) $m / z(\%): 245$ (7), 244 (50), 215 (39), 187 (35), 170 (100), 159 (44), 142 (40), 141 (54), 94 (25), 77 (70).

(4-甲基苯基)膦酸二乙酯(3e) ${ }^{[18]}$ : 无色油状物. ${ }^{1} \mathrm{H}$ NMR $\left(500 \mathrm{MHz}, \mathrm{CDCl}_{3}\right) \delta: 7.70(\mathrm{dd}, J=13.0,7.5 \mathrm{~Hz}$, $2 \mathrm{H}), 7.28 \sim 7.26(\mathrm{~m}, 2 \mathrm{H}), 4.17 \sim 4.02(\mathrm{~m}, 4 \mathrm{H}), 2.40(\mathrm{~s}$, $3 \mathrm{H}), 1.31$ (t, $J=7.0 \mathrm{~Hz}, 6 \mathrm{H}) ;{ }^{31} \mathrm{P}$ NMR $(202 \mathrm{MHz}$, $\mathrm{CDCl}_{3}$ ) $\delta:$ 19.6; MS (EI) $m / z$ (\%): 229 (3), 228 (24), 172 (100), 156 (42), 155 (49), 119 (41), 108 (23), 92 (46), 91 (88), 65 (35).

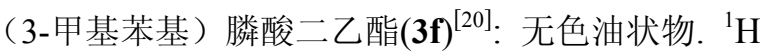
NMR (500 MHz, $\left.\mathrm{CDCl}_{3}\right) \delta: 7.65 \sim 7.58(\mathrm{~m}, 2 \mathrm{H}), 7.38 \sim$ $7.33(\mathrm{~m}, 2 \mathrm{H}), 4.19 \sim 4.03(\mathrm{~m}, 4 \mathrm{H}), 2.40(\mathrm{~s}, 3 \mathrm{H}), 1.33(\mathrm{t}$, $J=7.0 \mathrm{~Hz}, 6 \mathrm{H}) ;{ }^{31} \mathrm{P}$ NMR $\left(202 \mathrm{MHz}, \mathrm{CDCl}_{3}\right) \delta: 19.3$; MS (EI) m/z (\%): 229 (4), 228 (27), 182 (24), 172 (97), 156 (42), 155 (48), 119 (39), 92 (59), 91 (100), 65 (38).

(2-甲基苯基)膦酸二乙酯 $(\mathbf{3 g})^{[8]}$ : 无色油状物. ${ }^{1} \mathrm{H}$ $\operatorname{NMR}\left(500 \mathrm{MHz}, \mathrm{CDCl}_{3}\right) \delta: 7.91 \sim 7.87(\mathrm{~m}, 1 \mathrm{H}), 7.28 \sim$ $7.24(\mathrm{~m}, 3 \mathrm{H}), 4.18 \sim 4.03(\mathrm{~m}, 4 \mathrm{H}), 2.57(\mathrm{~s}, 3 \mathrm{H}), 1.32(\mathrm{t}$, $J=7.5 \mathrm{~Hz}, 6 \mathrm{H}) ;{ }^{31} \mathrm{P}$ NMR $\left(202 \mathrm{MHz}, \mathrm{CDCl}_{3}\right) \delta: 19.5$; MS (EI) $m / z$ (\%): 229 (8), 228 (66), 213 (27), 172 (100), 156 (46), 155 (45), 154 (71), 119 (61), 91 (88), 65 (33).

(4-氟苯基) 膦酸二乙酯 $(\mathbf{3 h})^{[21]}$ : 无色油状物. ${ }^{1} \mathrm{H}$ NMR $\left(500 \mathrm{MHz}, \mathrm{CDCl}_{3}\right) \delta: 7.85 \sim 7.80(\mathrm{~m}, 2 \mathrm{H}), 7.18 \sim$ $7.14(\mathrm{~m}, 2 \mathrm{H}), 4.19 \sim 4.04(\mathrm{~m}, 4 \mathrm{H}), 1.33(\mathrm{t}, J=7.0 \mathrm{~Hz}$, $6 \mathrm{H}) ;{ }^{31} \mathrm{P}$ NMR (202 MHz, $\mathrm{CDCl}_{3}$ ) $\delta$ : 17.8; MS (EI) $\mathrm{m} / \mathrm{z}$ (\%): 233 (2), 232 (20), 176 (100), 160 (53), 159 (80), 123 
(40), 112 (47), 96 (52), 77 (28), 65 (16).

(4-氯苯基) 膦酸二乙酯 $(3 \mathbf{i})^{[22]}$ : 无色油状物. ${ }^{1} \mathrm{H}$ $\operatorname{NMR}\left(500 \mathrm{MHz}, \mathrm{CDCl}_{3}\right) \delta: 7.77 \sim 7.75(\mathrm{~m}, 2 \mathrm{H}), 7.46 \sim$ $7.44(\mathrm{~m}, 2 \mathrm{H}), 4.19 \sim 4.04(\mathrm{~m}, 4 \mathrm{H}), 1.33(\mathrm{t}, J=7.0 \mathrm{~Hz}$, $6 \mathrm{H}) ;{ }^{31} \mathrm{P}$ NMR (202 MHz, $\mathrm{CDCl}_{3}$ ) $\delta: 17.6$; MS (EI) $\mathrm{m} / \mathrm{z}$ (\%): 249 (4), 248 (21), 192 (100), 176 (39), 175 (49), 139 (49), 128 (38), 112 (39), 75 (37), 65 (16).

(3-氯苯基) 膦酸二乙酯 $(\mathbf{3 j})^{[22]}$ : 无色油状物. ${ }^{1} \mathrm{H}$ NMR (500 MHz, $\left.\mathrm{CDCl}_{3}\right) \delta: 7.79(\mathrm{td}, J=14.0,1.5 \mathrm{~Hz}$, $1 \mathrm{H}), 7.72 \sim 7.67(\mathrm{~m}, 1 \mathrm{H}), 7.54 \sim 7.52(\mathrm{~m}, 1 \mathrm{H}), 7.44 \sim$ $7.40(\mathrm{~m}, 1 \mathrm{H}), 4.20 \sim 4.06(\mathrm{~m}, 4 \mathrm{H}), 1.34(\mathrm{t}, J=7.0 \mathrm{~Hz}$, $6 \mathrm{H}) ;{ }^{31} \mathrm{P} \mathrm{NMR}\left(202 \mathrm{MHz}, \mathrm{CDCl}_{3}\right) \delta: 16.5$; MS (EI) $\mathrm{m} / \mathrm{z}$ (\%): 249 (5), 248 (26), 194 (35), 192 (100), 175 (48), 139 (37), 128 (29), 112 (50), 75 (42), 65 (19).

(2-氯苯基)膦酸二乙酯 $(\mathbf{3 k})^{[22]}$ : 无色油状物. ${ }^{1} \mathrm{H}$ NMR (500 MHz, $\left.\mathrm{CDCl}_{3}\right) \delta: 8.01(\mathrm{dd}, J=14.0,7.5 \mathrm{~Hz}$, $1 \mathrm{H}), 7.49 \sim 7.44(\mathrm{~m}, 2 \mathrm{H}), 7.37 \sim 7.34(\mathrm{~m}, 1 \mathrm{H}), 4.25 \sim$ $4.10(\mathrm{~m}, 4 \mathrm{H}), 1.36(\mathrm{t}, J=7.0 \mathrm{~Hz}, 6 \mathrm{H}) .{ }^{31} \mathrm{P}$ NMR $(202$ $\mathrm{MHz}, \mathrm{CDCl}_{3}$ ) $\delta: 14.5$; MS (EI) $m / z(\%): 249$ (2), 248 (6), 213 (100), 192 (45), 185 (81), 175 (54), 139 (67), 112 (47), 75 (37), 65 (22).

(4-三氟甲基苯基)膦酸二乙酯 $(31)^{[23]}$ : 无色油状物. ${ }^{1} \mathrm{H}$ NMR (500 MHz, $\mathrm{CDCl}_{3}$ ) $\delta: 7.95$ (dd, $J=13.0,8.0$ $\mathrm{Hz}, 2 \mathrm{H}), 7.75 \sim 7.72(\mathrm{~m}, 2 \mathrm{H}), 4.23 \sim 4.08(\mathrm{~m}, 4 \mathrm{H}), 1.34$ $(\mathrm{t}, J=7.0 \mathrm{~Hz}, 6 \mathrm{H}) ;{ }^{31} \mathrm{P} \mathrm{NMR}\left(202 \mathrm{MHz}, \mathrm{CDCl}_{3}\right) \delta: 16.3$; MS (EI) $m / z(\%): 283$ (3), 282 (21), 255 (26), 227 (100), 210 (40), 209 (78), 162 (35), 145 (49), 110 (33), 65 (15).

(4-氧基苯基)膦酸二乙酯 $(\mathbf{3 m})^{[24]}$ : 无色油状物. ${ }^{1} \mathrm{H}$ NMR (500 MHz, $\left.\mathrm{CDCl}_{3}\right) \delta: 7.93(\mathrm{dd}, J=13.5,8.5 \mathrm{~Hz}$, $2 \mathrm{H}), 7.78 \sim 7.76(\mathrm{~m}, 2 \mathrm{H}), 4.23 \sim 4.09(\mathrm{~m}, 4 \mathrm{H}), 1.35(\mathrm{t}$, $J=7.0 \mathrm{~Hz}, 6 \mathrm{H}) ;{ }^{31} \mathrm{P} \mathrm{NMR}\left(202 \mathrm{MHz}, \mathrm{CDCl}_{3}\right) \delta: 15.3$; MS (EI) $m / z(\%): 240$ (4), 239 (18), 212 (26), 184 (100), 167 (45), 166 (85), 130 (47), 119 (40), 103 (46), 75 (14).

(4-乙酰基苯基)膦酸二乙酯 $(3 n)^{[17]}$ : 无色油状物. ${ }^{1} \mathrm{H}$ NMR $\left(500 \mathrm{MHz}, \mathrm{CDCl}_{3}\right) \delta: 8.04 \sim 8.02(\mathrm{~m}, 2 \mathrm{H})$, $7.92 \sim 7.89(\mathrm{~m}, 2 \mathrm{H}), 4.21 \sim 4.07(\mathrm{~m}, 4 \mathrm{H}), 2.63(\mathrm{~s}, 3 \mathrm{H})$, $1.33(\mathrm{t}, J=7.0 \mathrm{~Hz}, 6 \mathrm{H}) ;{ }^{31} \mathrm{P} \mathrm{NMR}\left(202 \mathrm{MHz}, \mathrm{CDCl}_{3}\right) \delta$ : 19.8; MS (EI) m/z (\%): 257 (3), 256 (22), 241 (54), 213 (100), 185 (58), 167 (33), 148 (31), 139 (19), 77 (21), 65 (23).

1-菜基膦酸二乙酯 $(\mathbf{3 0})^{[17]}$ : 无色油状物. ${ }^{1} \mathrm{H}$ NMR $\left(500 \mathrm{MHz}, \mathrm{CDCl}_{3}\right) \delta: 8.52(\mathrm{~d}, J=8.5 \mathrm{~Hz}, 1 \mathrm{H}), 8.25(\mathrm{dd}$, $J=16.0,7.0 \mathrm{~Hz}, 1 \mathrm{H}), 8.04$ (d, $J=8.0 \mathrm{~Hz}, 1 \mathrm{H}), 7.89$ (d, $J=8.0 \mathrm{~Hz}, 1 \mathrm{H}), 7.61(\mathrm{t}, J=7.5 \mathrm{~Hz}, 1 \mathrm{H}), 7.56 \sim 7.51(\mathrm{~m}$, $2 \mathrm{H}), 4.25 \sim 4.04(\mathrm{~m}, 4 \mathrm{H}), 1.31(\mathrm{t}, J=7.0 \mathrm{~Hz}, 6 \mathrm{H}) ;{ }^{31} \mathrm{P}$
NMR (202 MHz, $\left.\mathrm{CDCl}_{3}\right) \delta: 19.2 ; \mathrm{MS}(\mathrm{EI}) m / z$ (\%): 265 (7), 264 (49), 235 (38), 191 (18), 155 (94), 154 (31), 128 (100), 127 (64), 115 (37), 77 (15).

\section{References and note}

[1] (a) Quin, L. D. A Guide to Organophosphorus Chemistry, Wiley-Interscience, New York, 2000.

(b) Sasaki, M. In Chirality in Agrochemicals, Eds.: Kurihara, N.; Miyamoto, J.; Wiley \& Sons, Chichester, 1998, p. 85.

(c) Imamoto, T. In Handbook of Organophosphorus Chemistry, Ed.: Engel, R., Marcel Dekker, New York, 1992, Chapter 1.

(d) Crepy, K. V. L.; Imamoto, T. Top. Curr. Chem. 2003, $229,1$.

(e) Giorgio, C.; Gianmauro, O.; Gerard, A. P. Tetrahedron 2003, $59,9471$.

(f) Tang, W.; Zhang, X. Chem. Rev. 2003, 103, 3029.

[2] (a) Xu, Q.; Han, L.-B. Sci. Sinica Chim. 2010, 40, 802 (in Chinese). (徐清, 韩立彪, 中国科学: 化学, 2010, 40, 802.)

(b) Xu, Q.; Han, L.-B. J. Organomet. Chem. 2011, 696, 130.

(c) Xu, Q.; Zhao, C.-Q.; Zhou, Y.-B.; Yin, S.-F.; Han, L.-B. Chin. J. Org. Chem. 2012, 32, 1761 (in Chinese).

(徐清, 赵长秋, 周永波, 尹双风, 韩立彪, 有机化学, 2012, 32, 1761.)

[3] (a) Schwan, A. L. Chem. Soc. Rev. 2004, 33, 218.

(b) Glueck, D. S. Coord. Chem. Rev. 2008, 252, 2171.

(c) Prim, D.; Campagne, J.; Joseph, D.; Andrioletti, B. Tetrahedron 2002, 58, 2041.

(d) Beletskaya, I. P.; Kazankova, M. Russ. J. Org. Chem. 2002, 38, 1391.

(e) Engel, R.; Cohen, J. I. Synthesis of Carbon-Phosphorus Bonds, 2nd ed., CRC Press, Boca Raton, 2003.

[4] (a) Han, L.-B.; Tilley, T. D. J. Am. Chem. Soc. 2006, 128, 13698. (b) Han, L.-B.; Ono, Y.; Shimada, S. J. Am. Chem. Soc. 2008, 130, 2752.

(c) Gao, Y.; Wang, G.; Chen, L.; Xu, P.; Zhao, Y.; Zhou, Y.; Han, L.-B. J. Am. Chem. Soc. 2009, 131, 7956.

(d) Zhou, Y.; Yin, S.; Gao, Y.; Zhao, Y.; Goto, M.; Han, L.-B. Angew. Chem., Int. Ed. 2010, 49, 6852.

(e) Xu, Q.; Shen, R.; Ono, Y.; Nagahata, R., Shimada, S.; Goto, M.; Han, L.-B. Chem. Commun. 2011, 47, 2333.

(f) Chen, Y.-R.; Duan, W.-L. J. Am. Chem. Soc. 2013, 135, 16754. (g) Feng, C.-G.; Ye, M.; Xiao, K.-J.; Li, S.; Yu, J.-Q. J. Am. Chem. Soc. 2013, 135, 9322.

(h) Unoh, Y.; Hirano, K.; Satoh, T.; Miura, M. Angew. Chem., Int. Ed. 2013, 52, 12975.

[5] (a) Miyaura, N.; Suzuki, A. Chem. Rev. 1995, 95, 2457.

(b) Miyaura, N. Top. Curr. Chem. 2001, 219, 11.

(c) Candeias, N. R.; Montalbano, F.; Cal, P. M. S. D.; Gois, P. M. P. Chem. Rev. 2010, 110, 6169.

(d) Partyka, D. V. Chem. Rev. 2011, 111, 1529.

(e) Chan, D. M. T.; Lam, P. Y. S. In Boronic Acids, Ed.: Hall, D. G., Wiley-VCH, Weinheim, 2005, Chaper 5, p. 205.

(f) Xiao, J. X.; Lam, P. Y. S. Synthesis 2011, 829.

[6] (a) Chan, D. M. T.; Monaco, K. L.; Wang, R.-P.; Winters, M. P. Tetrahedron Lett. 1998, 39, 2933.

(b) Lam, P. Y. S.; Clark, C. G.; Saubern, S.; Adams, J.; Winters, M. P.; Chan, D. M. T.; Combs, A. Tetrahedron Lett. 1998, 39, 2941.

(c) Evans, D. A.; Katz, J. L.; West, T. R. Tetrahedron Lett. 1998, 39, 2937.

[7] (a) Theil, F. Angew. Chem., Int. Ed. 1999, 38, 2345.

(b) Collman, J. P.; Zhong, M.; Zhang, C.; Costanzo, S. J. Org. 
Chem. 2001, 66, 7892

(c) Rao, H.; Fu, H.; Jiang, Y.; Zhao, Y. Angew. Chem., Int. Ed. 2009, 48, 1114.

(d) Xu, H.-J.; Zhao, Y.-Q.; Feng, T.; Feng, Y.-S. J. Org. Chem. 2012, 77, 2878.

(e) Sueki, S.; Kuninobu, Y. Org. Lett. 2013, 15, 1544.

(f) Yang, M.; Pei, J.; Yan, G.; Weng, Q. Chin. J. Org. Chem. 2013, 33, 343 (in Chinese).

(杨明华, 裴吉, 严国兵, 翁秋月, 有机化学, 2013, 33, 343.)

(g) Taniguchi, N. J. Org. Chem. 2007, 72, 1241.

(h) Yu, J.-T.; Guo, H.; Yi, Y.; Fei, H.; Jiang, Y. Adv. Synth. Catal. 2014, 356, 749 .

[8] Andaloussi, M.; Lindh, J.; Sävmarker, J.; Sjöerg, P. J. R.; Larhed, M. Chem. Eur. J. 2009, 15, 13069.

[9] (a) Zhuang, R.; Xu, J.; Cai, Z.; Tang, G.; Fang, M.; Zhao, Y. Org. Lett. 2011, 13, 2110.

(b) Hu, G.; Chen, W.; Fu, T.; Peng, Z.; Qiao, H.; Gao, Y.; Zhao, Y. Org. Lett. 2013, 15, 5362.

[10] (a) Ley, S. V.; Thomas, A. W. Angew. Chem., Int. Ed. 2003, 42, 5400 .

(b) Evano, G.; Blanchard, N.; Toumi, M. Chem. Rev. 2008, 108, 3054 .

(c) Ma, D.; Cai, Q. Acc. Chem. Res. 2008, 41, 1450.

(d) Monnier, F.; Taillefer, M. Angew. Chem., Int. Ed. 2009, 48, 6954.

[11] Allen, S. E.; Walvoord, R. R.; Padilla-Salinas, R.; Kozlowski, M. C. Chem. Rev. 2013, 113, 6234 and references therein.

[12] (a) Tian, H.; Yu, X.; Li, Q.; Wang, J.; Xu, Q. Adv. Synth. Catal. 2012, 354, 2671. (b) Huang, B.; Tian, H.; Lin, S.; Xie, M.; Yu, X.; Xu, Q. Tetrahedron Lett. 2013, 54, 2861.

[13] During the progress of our present work, Zhao and coworkers reported similar $\mathrm{Cu}$ - and $\mathrm{Ni}$-catalyzed $\mathrm{C}-\mathrm{P}$ coupling reactions of arylboronic acids with $\mathrm{P}-\mathrm{H}$ compounds (see Ref. [9]).

[14] Ouyang, K.; Xi, Z. Acta Chim. Sinica 2013, 71, 13 (in Chinese). (欧阳昆冰, 席振峰, 化学学报, 2013, 71, 13.)

[15] (a) Kirai, N.; Yamamoto, Y. Eur. J. Org. Chem. 2009, 1864.

(b) Cheng, G.; Luo, M. Eur. J. Org. Chem. 2011, 2519.

(c) Mao, J.; Hua, Q.; Xie, G.; Yao, Z.; Shi, D. Eur. J. Org. Chem. 2009, 2262.

[16] (a) Zhao, B.; Lu, X. Tetrahedron Lett. 2006, 47, 6765.

(b) Bouffard, J.; Itami, K. Org. Lett. 2009, 11, 4410.

(c) Wong, Y.-C.; Parthasarathy, K.; Cheng, C.-H. Org. Lett. 2010, 12, 1736.

(d) Liao, Y.-X.; Xing, C.-H.; Hu, Q.-S. Org. Lett. 2012, 14, 1544.

[17] Luo, Y.; Wu, J. Organometallics 2009, 28, 6823.

[18] Ma, M.; Peng, Z.; Chen, L. Chin. J. Chem. 2006, 24, 1391.

[19] Bonnaventure, I.; Charette, A. B. J. Org. Chem. 2008, 73, 6330.

[20] Kagayama, T.; Nakano, A.; Sakaguchi, S. Org. Lett. 2006, 8, 407.

[21] Kalek, M.; Jezowska, M.; Stawinski, J. Adv. Synth. Catal. 2009, $351,3207$.

[22] Berrino, R.; Cacchi, S.; Fabrizi, G. Org. Biomol. Chem. 2010, 8, 4518.

[23] Bennett, J. A.; Hope, E. G.; Singh, K.; Stuart, A. M. J. Fluorine Chem. 2009, 130, 615.

[24] Kohler, M. C.; Sokol, J. G.; Stockland, R. A. Tetrahedron Lett. 2009, 50, 457 . 